subsequently diagnosed with TBM and analyses the impact of CPAP on exacerbations caused by TBM.

Methods 20 TBM patients were identified at the North West Lung Centre in University Hospital South Manchester (UHSM) through clinics and department databases. Patient case notes and general practice medication prescriptions were used to obtain data on antibiotic and steroid prescriptions as well as hospital admissions (one year before and one after treatment). Exacerbations were analysed pre- and post-CPAP. Patient demographics were also recorded and compared to the cohort in the national severe asthma registry.

Results TBM patients were found to have increased weight and BMI compared to the cohort described in the national severe asthma registry; weight $92.4 \pm 28.8$ in TBM compared to $81.2 \pm 19.9$ and BMI $35.5 \pm 8.1$ compared to 28.0. Analysis of CPAP data showed that with an average of 6 hours of CPAP daily at average pressures of $11 \mathrm{~cm} \mathrm{H}_{2} \mathrm{O}$ significantly reduced the number of annual exacerbations experienced by over $50 \%$ for antibiotic and steroid prescriptions and significantly more for hospital admissions. Figure 1 shows the reduction in exacerbations in the year following the initiation of CPAP compared to the year before.

Conclusions The severe asthma cohort with TBM are likely to have an increased weight and BMI. CPAP is an effective treatment option for TBM patients whilst awaiting the availability of more definitive surgical options and reduces antibiotic and steroid prescriptions as well as hospital admissions. It is recommended that all symptomatic TBM patients are considered for CPAP and monitored for exacerbations thereafter.

\section{S55 USING CONTINUOUS POSITIVE AIRWAY PRESSURE (CPAP) IN EXCESSIVE DYNAMIC AIRWAY COLLAPSE (EDAC)}

AP Hicks, T Brown, A Chauhan, K Adeniji, M Quint, S Babu. Queen Alexandra Hospital, Romsey, UK

\subsection{6/thoraxjnl-2016-209333.61}

EDAC is a term that refers to the pathological collapse of respiratory airways during expiration as a result of posterior wall muscle laxity leading to a $>50 \%$ loss of airway cross-sectional area. This muscle laxity leads to a loss of airway patency which results in symptomatic dyspnoea. CPAP has been suggested as a method to ameliorate the difficulties associated with EDAC where standard medical care has failed, ameliorating the need for further invasive treatments such as endobronchial stenting or tracheoplasty. Demonstrating its effectiveness is difficult as many of those who suffer with EDAC have co-morbid disease such as COPD, asthma or EGPA which may mask the impact of CPAP when measuring response with subjective criteria such as the WHO functional impairment scale. We set out to determine whether we could objectively demonstrate improvements in airway diameter using CPAP in patients with EDAC, in addition to COPD and EGPA, using firstly bronchoscopy and secondly dynamic computed tomography (CT). In both cases we used a Philips Respironics Trilogy 200 CPAP device to deliver positive airway pressure at 5 $\mathrm{cm}$ increments up to a pressure of $20 \mathrm{cmH} 2 \mathrm{O}$. Figure 1a shows a bronchoscopic example of this process with an increase in airway area from $1.54 \mathrm{~cm}^{2}$ to $5.35 \mathrm{~cm}^{2}$ equating to a $400 \%$ increase in volume at $20 \mathrm{~cm} \mathrm{H}_{2} \mathrm{O}$. Figure $1 \mathrm{~b}$ shows a CT example with a $52.9 \%$ increase in airway area, with the RMB increasing in diameter by $3 \mathrm{~mm}$. CPAP was objectively shown to be an effective,

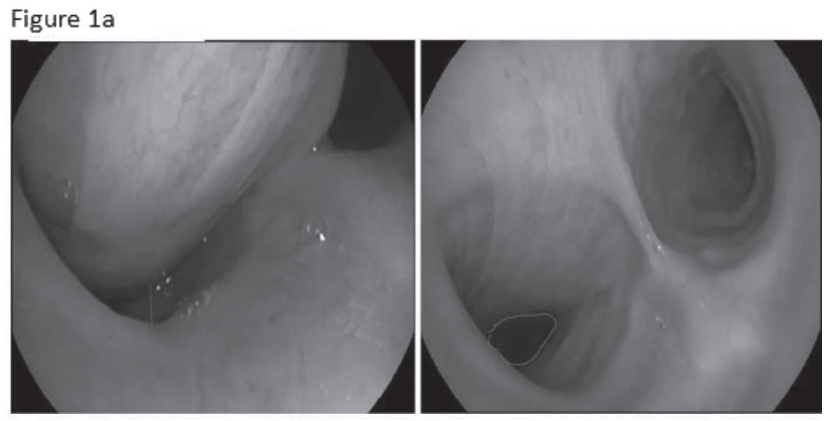

Figure $1 b$

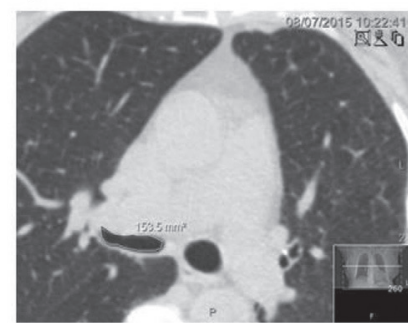

No CPAP

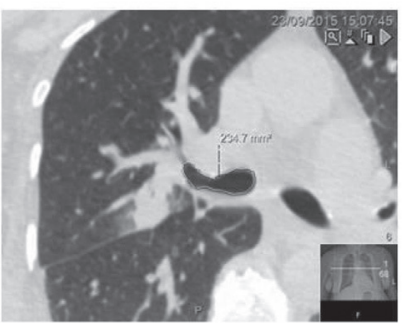

CPAP
Abstract S55 Figure 1

relatively inexpensive, treatment for EDAC via bronchoscopy or CT imaging and it is hypothesised such independent measures enhance existing assessments of improvement.

\section{S56 NEURAL RESPIRATORY DRIVE AND CARDIAC FUNCTION IN PATIENTS WITH OBESITY-HYPOVENTILATION- SYNDROME FOLLOWING SETUP OF NON-INVASIVE VENTILATION FOR HYPERCAPNIC RESPIRATORY FAILURE}

A Onofri, M Patout, G Arbane, M Pengo, P Marino, J Steier. Lane Fox Respiratory Unit, Guy's and St. Thomas' Foundation Trust, London, UK

\subsection{6/thoraxjnl-2016-209333.62}

Background Chronic hypercapnic respiratory failure in obesity hypoventilation syndrome (OHS) is commonly treated with noninvasive ventilation (NIV). We hypothesised that treatment of OHS would improve neural respiratory drive (NRD) and improve cardiac function.

Patients and methods A prospective, observational single-centre study was conducted. OHS patients were assessed recording $\mathrm{NRD}$, as measured by the electromyogram of the parasternal intercostals (EMGpara) before, during and after NIV set-up and cardiac function with trans-thoracic echocardiography (TTE) before and after NIV set-up. Follow up appointments were planned at 6-weeks (6W-FU) and 3 Months (3M-FU). The tricuspid annular plane systolic excursion (TAPSE) score was used to assess the right ventricular (RV) function and EMGpara\%max and neural respiratory drive index (NRDI) were recorded to assess NRD. The Wilcoxon test was used to compare baseline with follow-up results.

Results 10 patients (age 55.9 (7.6) years, females 50\%, weight $126.6(29.1) \mathrm{kg}$, BMI $\left.48.1(7.5) \mathrm{kg} / \mathrm{m}^{2}\right)$ were studied. 3 patients were non-compliant with NIV. NRDI and EMGpara\%maxsignificantly improved following NIV set-up, and this effect was maintained at 3M-FU (EMGpara\%max 24.4 (12.9)\%, 16.9 (5.4)\% 
and $18.6(6.5) \%, \mathrm{p}=0.028$ and $\mathrm{p}=0.035 ;$ NRDI 480.4 $(256.0) / \mathrm{min}, 314.7(125.6) / \mathrm{min}$ and $379.5(138.0) / \mathrm{min}, \mathrm{p}=0.22$ and $\mathrm{p}=0.012$; Figure 1$)$.

There were no significant differences in cardiac function between baseline and 3M-FU (TAPSE: 2.6 (0.6) mm vs. $2.4(0.4)$ $\mathrm{mm}, \mathrm{p}=1.00$ ) or systolic pulmonary artery pressures (sPAP 36.7 (15.2) $\mathrm{mmHg}$ vs 35.8 (16.2) $\mathrm{mmHg}$, p: 0.50). The TAPSE score in compliant patients seemed to improve $(\mathrm{n}=3 ; 2.3(0.6) \mathrm{mm}$ vs. $2.7(0.3) \mathrm{mm})$ while non compliant patients experienced a deterioration ( $\mathrm{n}=3 ; 2.7(0.5) \mathrm{mm}$ vs. $2.2(0.4) \mathrm{mm}$ ).

Conclusions NIV improves NRD and respiratory parameters in patients with OHS. However, cardiac function does not improve over a three-month period despite the significant improvements in ventilation. These results are influenced by treatment adherence.

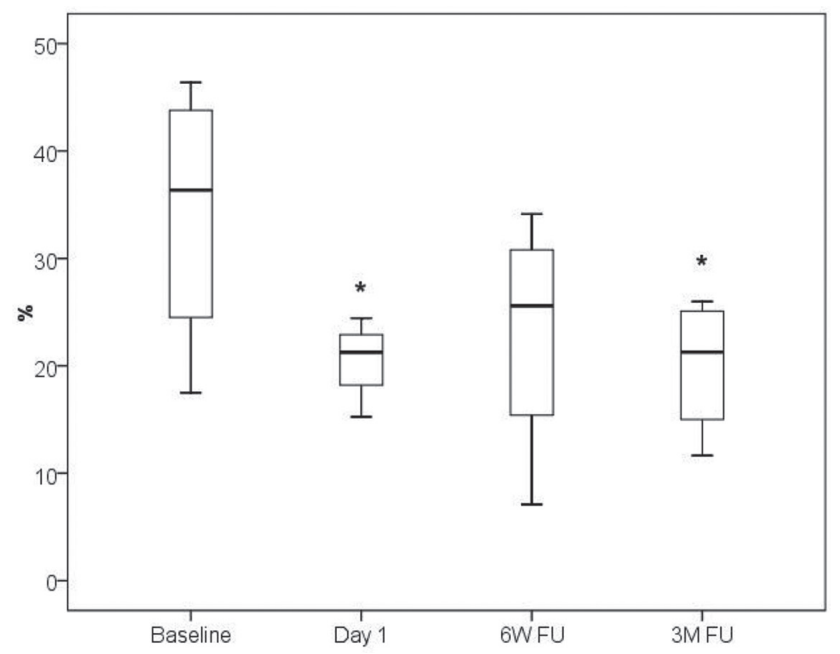

Abstract S56 Figure 1 EMG para\%max improves following setup of NIV And at 3 month in OHS: $\left({ }^{*}\right): p<0.05$

\section{S57 QUALITATIVE ASSESSMENT OF THE EXPERIENCE OF TELEMONITORING IN VENTILATED PATIENTS WITH MOTOR NEURONE DISEASE}

${ }^{1} \mathrm{H}$ Ashcroft, ${ }^{1} \mathrm{H}$ Ando, ${ }^{2} \mathrm{R}$ Halhead, ${ }^{1} \mathrm{~B}$ Chakrabarti, ${ }^{3} \mathrm{CA}$ Young, ${ }^{4} \mathrm{R}$ Cousins, ${ }^{1} \mathrm{RM}$ Angus. ${ }^{1}$ Aintree University Hospital NHS Foundation Trust, Liverpool, UK; ${ }^{2}$ Docobo Ltd, Leatherhead, UK; ${ }^{3}$ The Walton Centre NHS Foundation Trust, Liverpool, UK; ${ }^{4}$ Liverpool Hope University, Liverpool, UK

\subsection{6/thoraxjnl-2016-209333.63}

Background The National Institute for Health and care Excellence (NICE) has recently issued recommendations on the care of people with motor neurone disease (MND), promoting tailored care for each patient, Guideline 42, 2016. Previous studies suggest remote monitoring offers a facility to regularly monitor and interact with patients, providing timely interventions so it may facilitate delivery of the recommendations. The efficacy of this approach is dependent upon acceptability of telemonitoring to patients.

Aim To understand the experiences of using telemonitoring in ventilated patients with MND.

Methods Semi-structured interviews were conducted with seven patients (male $=5$; mean age $=63 \mathrm{yrs}$ ). The median illness duration was $14 \mathrm{~m}$ (range $=7 \mathrm{~m}-13$ yrs $7 \mathrm{~m}$ ) and the median non- invasive ventilation (NIV) usage was $12 \mathrm{~m}$ (range $=0 \mathrm{~m}-3 \mathrm{yrs}$ ). Participants used a telemonitoring device (Docobo CAREPOR$\mathrm{TAL}^{\circledR}$ ) for six months, completed weekly nocturnal pulse oximetry and symptom-related questions. Five caregivers were present at the interviews and provided their feedback. Interviews were audio recorded and transcribed verbatim. Thematic analysis was conducted to find overarching themes. The interpretation was reviewed and supported by a multidisciplinary team examination. Findings Five themes were identified: Technical Challenges, Increased Self-Awareness, Taking Initiative, Benefits of Timely Intervention, and Reducing the Unnecessary. Whilst participants expressed general ease of Careportal ${ }^{\circledR}$ use, technical issues included; messaging system challenges, oximetry transmission, device fault, mobile signal loss. No other negative experience of using Careportal ${ }^{\circledR}$ was reported. Overall, participants expressed how telemonitoring enabled symptom awareness and interpretation. The device also enabled the participants to raise their concerns and/or requests to the healthcare professionals via the messaging system, and this was depicted as a sharp contrast to current communication with hospitals. Timely interventions were observed as a result of regular monitoring, contributing to both physical and psychological well-being of the participants. It was also suggested that using Careportal ${ }^{\circledR}$ could reduce unnecessary cost/time and hassles created by attending hospital appointments. Conclusions Telemonitoring enabled participants to be actively involved in their care and they felt that the interventions were timely delivered to meet their needs. The findings suggest potential benefits of utilising Careportal ${ }^{\circledR}$ in routine care as a contact point to accommodate different individual's needs.

Supported by an SBRI Grant

\section{S58 THE USE OF REMOTE MONITORING TO ASSESS VENTILATOR ADHERENCE AND OUTCOMES WITHIN A REGIONAL HOME MECHANICAL VENTILATION SERVICE}

${ }^{1}$ YM Gn, ${ }^{2} \mathrm{R}$ Moses, ${ }^{2} \mathrm{~A}$ Vyas. ${ }^{1}$ Manchester Medical School, Manchester, UK; ${ }^{2}$ Lancashire Teaching Hospitals NHS Foundation Trust, Preston, UK

\subsection{6/thoraxjnl-2016-209333.64}

Introduction Home mechanical ventilation (HMV) is a recognised evidence-based intervention for patients in chronic respiratory failure (CRF). However there is a paucity of evidence on the adherence to this treatment. This study examined the adherence of HMV and evaluated the clinical outcomes associated with adherence in a variety of patient populations - namely neuromuscular disease, chest wall disease, obesity hypoventilation syndrome and chronic obstructive pulmonary disease (COPD).

Methods A retrospective study was carried out between May to June 2016. Adherence data was downloaded through remote monitoring. Primary outcomes included changes in blood gases at 3 and 6 months, and hospital admissions 12 months pre- and post-ventilation. Qualitative outcomes were obtained through a questionnaire conducted via telephone interviews.

Results 62 patients were included in this study. Patients undertaking remote monitoring of HMV demonstrated an adherence rate of $90.3 \%$, defined as more than 4 hours of ventilator use/night (mean $[\mathrm{SD}]=7 \mathrm{~h} 17 \mathrm{~min}[2 \mathrm{~h} 53 \mathrm{~min}])$. No significant difference in adherence was found between patients of different aetiologies. The blood gases improved significantly at 3 months compared to baseline $(\mathrm{p}<0.05)$. However, there was a universal trend for these parameters to return to baseline at 6 months regardless of the underlying disease. HMV reduced hospital admissions by 